\title{
Assessment of water vapor content from MIVIS TIR data
}

\author{
Cristiana Bassani $\left({ }^{1}\right)$, Vincenzo Cuomo $\left({ }^{1}\right)$, Vito Lanorte $\left({ }^{1}\right)$, Stefano Pignatti $\left({ }^{1}\right)\left({ }^{2}\right)$ and Valerio Tramutoli $\left({ }^{3}\right)$ \\ (') Istituto di Metodologie per l'Analisi Ambientale (IMAA), CNR, Tito Scalo (PZ), Italy \\ $\left(^{2}\right)$ Laboratorio Aereo Ricerche Ambientali (LARA), IIA-CNR, Tor Vergata (RM), Italy \\ $\left.{ }^{3}\right)$ Dipartimento di Ingegneria e Fisica dell'Ambiente (DIFA), \\ Università degli Studi della Basilicata, Potenza Italy
}

\begin{abstract}
The main objective of land remotely sensed images is to derive biological, chemical and physical parameters by inverting sample sets of spectral data. For the above aim hyperspectral scanners on airborne platform are a powerful remote sensing instrument for both research and environmental applications because of their spectral resolution and the high operability of the platform. Fine spectral information by MIVIS (airborne hyperspectral scanner operating in 102 channels ranging from VIS to TIR) allows researchers to characterize atmospheric parameters and their effects on measured data which produce undesirable features on surface spectral signatures. These effects can be estimated (and remotely sensed radiances corrected) if atmospheric spectral transmittance is known at each image pixel. Usually ground-based punctual observations (atmospheric sounding balloons, sun photometers, etc.) are used to estimate the main physical parameters (like water vapor and temperature profiles) which permit us to estimate atmospheric spectral transmittance by using suitable radiative transfer model and a specific (often too strong) assumption which enable atmospheric properties measured only in very few points to be extended to the whole image. Several atmospheric gases produce observable absorption features, but only water vapor strongly varies in time and space. In this work the authors customize a self-sufficient «split-window technique» to derive (at each image pixel) atmospheric total columnar water vapor content (TWVC) using only MIVIS data collected by the fourth MIVIS spectrometer (Thermal Infrared band). MIVIS radiances have been simulated by means of MODTRAN4 radiative transfer code and the coefficients of linear regression to estimate TWVC from «split-windows» MIVIS radiances, based on 450 atmospheric water vapor profiles obtained by radiosonde data provided by NOAAINESDIS. The method has been applied to produce maps describing the spatial variability of the water vapor columnar content along a trial scene. The procedure has been validated by means of the MIVIS data acquired over Venice and the contemporary radiosonde data. A discrepancy within 5\% has been measured between the estimate of TWVC derived from the proposed self-sufficient split-window technique and the coincident radiosonde measurements. If confirmed by further analyses such a result will permit us to fully exploit MIVIS TIR capability to offer a more effective (at image pixel level) and self-sufficient (no ancillary observations required) way to obtain atmospherically corrected MIVIS radiances.
\end{abstract}

Key words radiative transfer code - water vapor split windows - MIVIS - atmospheric correction

Mailing address: Dr. Cristiana Bassani, Istituto di Metodologie per l'Analisi Ambientale (IMAA), CNR, C.da S. Loja, 85050 Tito Scalo (PZ), Italy; e-mail: bassani@imaa.cnr.it

\section{Introduction}

The parameterization of variables defining the interaction between soil and atmosphere radiances plays an important role to define an operative methodology to characterize soil spectral properties over wide surfaces through MIVIS (Multispectral Infrared and Visible Imaging Spectrometer) optical hyperspectral data. 
Atmospheric absorption and scattering processes by atmospheric constituents (mainly water vapor, carbon dioxide, oxygen and ozone) and aerosols, invalidate the interpretability of data to various degrees because of their interference with the energy at the sensor level.

The correction of radiance values at the sensor level has been resolved by applying correction methods based on algorithms (like 6S, Tanré et al., 1986; ATREM, Gao and Goetz, 1990; ACORN, AIG, 2001, etc.), which enable atmospheric profiles (altitude, pressure, temperature, water vapor and ozone) and both standard and user-defined aerosol models to be managed. Among all the parameters determining the revision process of the radiance measured at the sensor, water vapor plays the most important part as it is the foremost element for thermodynamics of the atmosphere. Its very high spatial and time variability makes the water vapor measurement derived from a single meteorological balloon or ground station inadequate whenever these data are to be used for correcting scenes covering several kilometers. From this point of view, the definition of a spectralbased water vapor columnar content evaluation at image pixel level can assure better prospects.

This paper proposes a split-window technique (following the approach proposed by Kleespies and McMillin, 1984) which establish- es a linear relation between the radiances measured in two TIR MIVIS channels and the Total Columnar Water Vapor Content (TCWVC) in the atmosphere. The coefficients of the linear multiple regression are derived from synthetic MIVIS radiances and an experimental dataset of 450 radiosonde water vapor profiles supplied by NOAA-NESDIS (National Environmental Satellite, Data and Information Service, www.nesdis.noaa.gov). The proposed method was validated using real MIVIS radiances measured during a flight over the Venice Lagoon to produce a (pixel by pixel) water vapor map over the whole scene and to give a first estimate of its accuracy by comparison with TCWVC achieved by contemporary radiosonde data.

\section{Measurement of the water vapor columnar content}

In order to correct data collected by different hyperspectral sensors (in particular AVIRIS), the three channel rationing method proposed by Gao and Goetz (1990) was implemented in the ATREM atmospheric correction code. It enables the TCWVC to be retrieved on a pixel by pixel basis using radiances measured in the water vapor absorption bands around $0.94 \mu \mathrm{m}$ and 1.14 $\mu \mathrm{m}$. This technique makes it possible to attain a

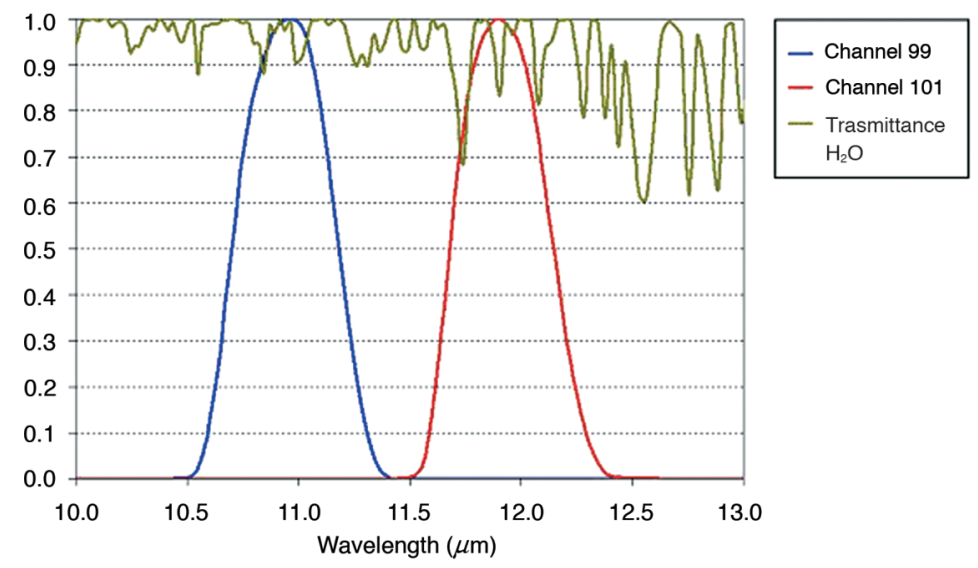

Fig. 1. MIVIS channel 99 (blue) and 101 (red) response functions versus trasmittance (green). 
good assessment of the water vapor columnar content if $0.94 \mu \mathrm{m}$ and $1.14 \mu \mathrm{m}$ channels are used. It is then inapplicable to the MIVIS (which does not have the same spectral capability) and inefficacious when employed in the NIR MIVIS channels sounding in the water vapor absorption bands at $0.72 \mu \mathrm{m}$ and/or $0.82 \mu \mathrm{m}$.

The split-window technique described by Keesplies and McMillin (1984), Jedlovec (1990), Ottle et al. (1997) and Menzel and Gumley (1998) instead defines the structure of a linear relation between the atmospheric TCWVC and radiances collected in the water vapor absorption bands in the 8-13 $\mu \mathrm{m}$ Thermal Infra-Red (TIR) spectral region. This region is well covered by the MIVIS with 10 channels (within 8.2 and 12.7 $\mu \mathrm{m})$. Figure 1 shows the spectral response of the MIVIS channels 99 and 101 versus water vapor transmittance. The MIVIS band 99 centered at 11 $\mu \mathrm{m}$ corresponds to a window region for water vapor, while the band 101 centered at $12 \mu \mathrm{m}$ intercepts small water vapor absorption peaks. The combination of the 99 and 101 MIVIS bands allows the water vapor columnar content to be solved by means of a split window technique according to Menzel's theory. In the atmospheric window from 8 to $14 \mu \mathrm{m}$ the water absorption is so low that the Taylor series expansion of the atmospheric transmittance $\left(\tau_{w}\right)$ could be approximate to the first-order

$$
\tau_{w}=\mathrm{e}^{-K_{w} u}=1-K_{w} u
$$

where $u$ and $K_{w}$ are respectively the total optical atmospheric column absorption path length due to the water vapor and the extinction coefficient at the absorbing water vapor wavelength $(w)$ which is supposed constant from the ground to the sensor altitude.

Under this assumption, an infinitesimal variation of $\tau_{w}$ is expressed

$$
d \tau_{w}=-K_{w} d u .
$$

In clear sky conditions the radiance received at the sensor at wavelength $w$ is the sum of the radiance contributions from the Earth's surface (first addendum of eq. (2.3)) and from all levels in the atmosphere (second addendum of eq. (2.3)) until sensor level as follows (Schwarzschild's equation)

$$
R_{w}=B_{w}^{S}\left(1-K_{w} u_{s}\right)+K_{w} \int_{0}^{u_{s}} B_{w} d u
$$

where $u_{s}$ represents the total atmospheric column absorption path length due to water vapor, $B_{w}$ is the Planck radiance from all the atmosphere levels from sensor altitude to the surface at the $w$ wavelength, and $B_{w}^{s}$ expresses the black body radiance from the surface at the same wavelength.

Taking into consideration the Integral Medium Value theorem

$$
\bar{B}_{w}=\frac{\int_{0}^{u_{s}} B_{w} d u}{\int_{0}^{u_{s}} d u}
$$

eq. (2.3) could be expressed as follows:

$$
R_{w}=B_{w}^{s}\left(1-K_{w} u_{s}\right)+K_{w} \bar{B}_{w} u_{s} .
$$

As a consequence $u_{s}$ could be expressed as

$$
u_{s}=\frac{R_{w}-B_{w}^{s}}{K_{w}\left(\bar{B}_{w}-B_{w}^{s}\right)} .
$$

Setting $w$ equal both at 11 and $12 \mu \mathrm{m}$, respectively in a water vapor window and absorbing region spectrally close to it, it follows that:

$$
\begin{gathered}
u_{s}=\frac{R_{11}-B_{11}^{s}}{K_{11}\left(\bar{B}_{11}-B_{11}^{s}\right)} \\
u_{s}=\frac{R_{12}-B_{12}^{s}}{K_{12}\left(\bar{B}_{12}-B_{12}^{s}\right)} .
\end{gathered}
$$

Taking into consideration the split window approximation which allows $B_{11}^{s}=B_{12}^{s}$ to be set, the eqs. ((2.6) and (2.7)) could be expressed as function of $B_{11^{s}=B_{12}^{s}}$, therefore $1 / u_{s}$ is expressed as follows:

$$
\frac{1}{u_{s}}=a \frac{R_{11}}{R_{12}-R_{11}}+b \frac{R_{12}}{R_{12}-R_{11}}
$$

where $\bar{B}_{w}$ is assumed proportional to $B_{w}^{s}$ by means of the proportionality coefficient $a_{w}$

$$
\bar{B}_{w}=a_{w} B_{w}^{s}
$$


therefore

$$
\begin{aligned}
& a=K_{12} a_{12}-K_{12} \\
& b=K_{11}-K_{11} a_{12} .
\end{aligned}
$$

Consequently $a$ and $b$ are the coefficients to be estimated to assess the water vapor columnar content from eq. (2.8).

In the case of the MIVIS, the $R_{11}$ and $R_{12}$ radiances, which solve the contrast between the surface and the atmosphere close to it (Menzel and Gumley, 1998), correspond to the band 99 (centered at $11 \mu \mathrm{m}$ ) and the band 101 (centered at $12 \mu \mathrm{m})$.

The $a$ and $b$ (eqs. (2.10a) and (2.10b)) coefficients were derived by applying a Multiple Regression Analysis (MRA) to the synthetic MIVIS radiances obtained through be application of the radiative transfer code MODTRAN (Berk et al., $1989)$ to 450 NESDIS radiosonde profiles. In order to obtain the expected MIVIS radiances in the channels 99 and 101, the TIR radiances were estimated by MODTRAN (setting time and geographic location variables according to the real MIVIS scene under examination) and then integrated on the MIVIS 4th spectrometer's spectral response functions. The obtained data set simulates the radiances that the sensor would have if measured under the atmospheric condition of each NESDIS radio sounding.

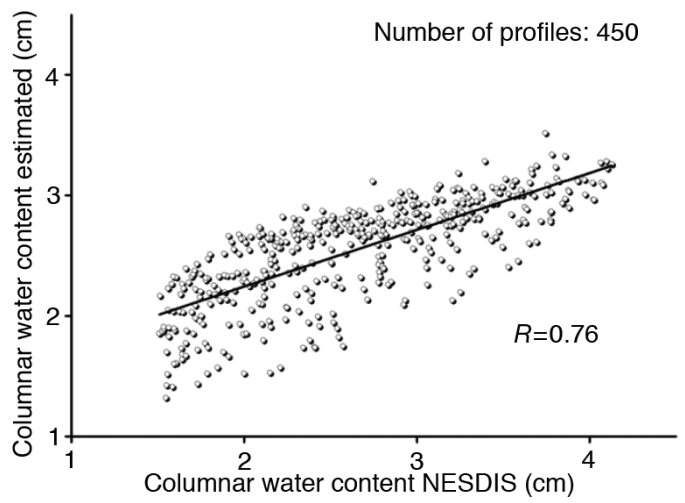

Fig. 2. Correlation index between the columnar content estimated and NESDIS.
Each NESDIS radiosounding was then used to obtain $1 / u_{s}$ in eq. (2.8) which determines an overestimated system of 450 linear equations. The radiances $R_{11}$ and $R_{12}$ simulated by MODTRAN, for each NESDIS profile, create the matrix of independent variables while real $u_{s}$ value, derived from the NESDIS observation, composes the vector of dependent variables.

The reliability of the MRA on this system made of 450 equations and two unknowns, $a$ and $b$ (eqs. (2.10a) and (2.10b)), is corroborated by the high value of the correlation index $(R=0.76)$ and by the relatively low value $(0.57$ $\mathrm{cm}$ ) of root mean square (fig. 2).

\section{Experimental data set}

The MIVIS flight performed on July 26, 2001 at 11:30 local time over the Venice Lagoon at $4000 \mathrm{~m}$ a.s.l. corresponding to a pixel ground resolution of $8 \mathrm{~m}$, was chosen to test the proposed method. The area selected for the test corresponds to the golf course of Malamocco where ground truth and atmospheric measurement were taken during the MIVIS image acquisition. The MIVIS flight line was converted into instrument perceiving radiance with an ad hoc IDL code running independently from the usual pre-processing chain that produces brightness temperature in the TIR region. Atmospheric profiling was achieved by means of a Vaisala radiosonde launched according to the airborne survey. The radiosonde atmospheric profiles (from surface up to $10000 \mathrm{~m}$ a.s.1.) were acquired in either the ascending and descending phase.

The radiances measured by the MIVIS channels 99 and 101 were employed (together with the $a$ e $b$ coefficients, previously determined) to estimate, on the basis of the eq. (2.8), the TCWVC at each image pixel.

The derived water vapor content map stresses how the described procedure does not introduce rough numerical errors, since all the values found over the scene $(4000 \times 755$ pixels $)$ are compatible and comparable with those used by the most common radiative transfer models which adopt atmospheric profiles ranging from 0 to $6 \mathrm{~cm}$ of precipitable water. As matter of fact the water vapor spatial histogram of the ex- 

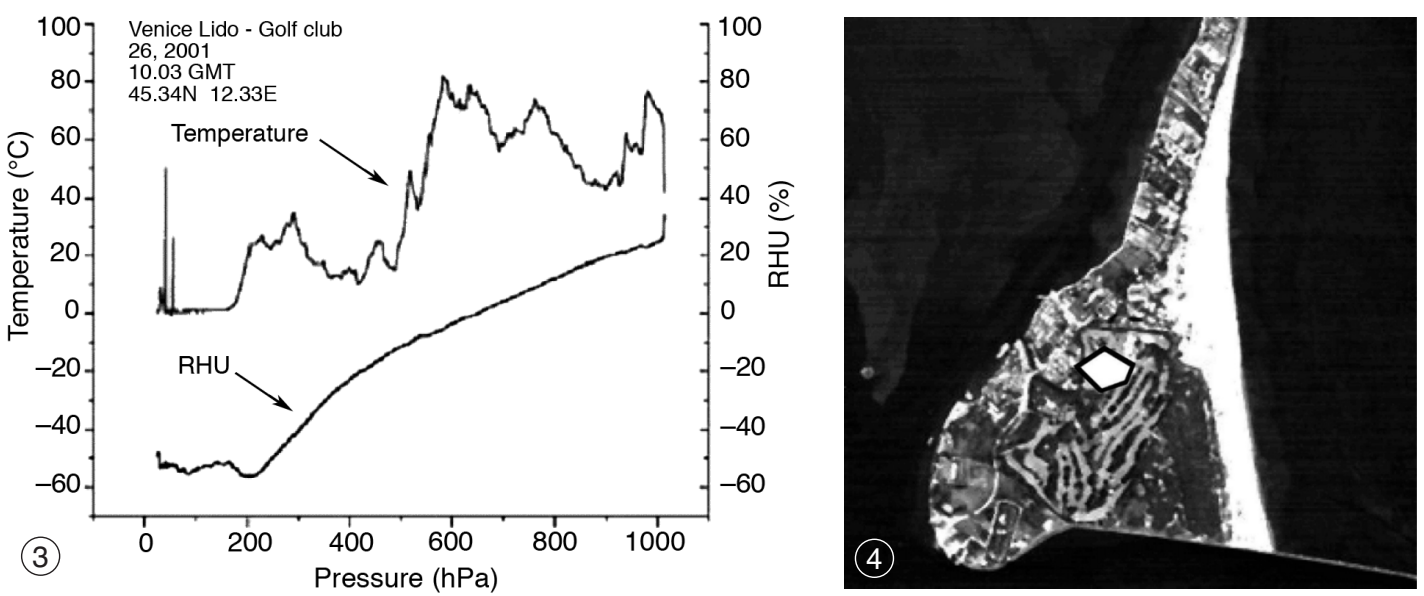

Fig. 3. Meteorological parameters profile, temperature and RHU (relative humidity) obtained with radiosounding balloon.

Fig. 4. Selected portion of interest (516 pixels) over radiosounding launched area (Malomocco, Lat: $45^{\circ} 72^{\prime} \mathrm{N}$; Long: $12^{\circ} 52^{\prime} \mathrm{W}$ ) into the nadir direction. The polygon is defined to apply the statistics of water vapor estimated by spectral-based method.

amined scene has a Gaussian shape centered at about $3 \mathrm{~cm}$ of water vapor with minimum value equal to $0.03 \mathrm{~cm}$ and maximum to $5.5 \mathrm{~cm}$. Such average spatial behavior of the TCWVC field is perfectly compatible with the measurement ( $\mathrm{TCWVC}=3.1 \mathrm{~cm}$ ) performed by the radiosonde launched at the same time of the MIVIS flight.

Selecting an Area Of Interest (AOI) of 516 pixels around the balloon launch area (fig. 3), into the nadir direction, the water vapor columnar content can vary with a range from $1.15 \mathrm{~cm}$ up to $3.63 \mathrm{~cm}$ with a mean value of $3.28 \mathrm{~cm}$. In this AOI of about $13000 \mathrm{~m}^{2}$ (comparable to a square with a side of more than $100 \mathrm{~m}$ ), over which the balloon was supposed to move during the ascending phase (fig. 4), the difference between the TCWVC value estimated by the proposed method and the one measured by the radiosonde is $0.16 \mathrm{~cm}$ only (about $5 \%$ ).

\section{Conclusions}

The procedure for water vapor assessment applied to the MIVIS images is based on a mul- tiple linear regression technique, connected to the application of a split window technique to the instrument's perceiving TIR radiance data. The MRA on the radio-sounding values of the NESDIS data base and MIVIS simulated radiances in the TIR region (channel 99 centered at $11 \mu \mathrm{m}$ and channel 101 centered at $12 \mu \mathrm{m}$ ) allowed the production of maps describing the spatial variability of the water vapor columnar content along the studied scene.

This procedure applied to the airborne hyperspectral data set acquired over the Venice Lagoon has given a difference of $0.16 \mathrm{~cm}(5 \%)$ with the TCWVC assessed by contemporary radiosounding.

It is clear that just a single MIVIS image cannot be considered statistically significant to affirm the exactness and validity of this procedure. Additional analyses will be carried out by using the MIVIS data collected during the campaigns in Sweden and in Sicily in June and July 2003. The several radiosoundings launched during these campaigns will be used to assess the reliability of this method in different landscapes. The data collected during the Sweden campaign will be particularly useful for this purpose, since the 
survey will be conducted within an international context and different hyperspectral airborne sensors will be employed. The trial will include pushbroom sensor covering the VNIR region and whiskbroom scanner like the MIVIS. Concomitant data acquisition in the VNIR region covering the $0.94 \mu \mathrm{m}$ water absorption bands (not present in the MIVIS spectral range) and in the TIR by MIVIS will allow a comparison of the straight line technique in the VNIR and the split window technique in the TIR. This experimental configuration based on the use of different methodologies to assess the water vapor columnar content will be an opportunity to better evaluate possible effects of surface material's spectral behaviour on the TCWVC estimate.

\section{Acknowledgements}

The authors are grateful to Angela Mirabelli for helping to improve the paper.

\section{REFERENCES}

Adler-Golden, S.M., M.W. Matthew, L.S. Bernstein, R.Y. LEVINE, A. BERK, S.C. RichtSMEIER, P.K. ACHARYA, G.P. Anderson, G. Felde, J. Gardner, M. Hike, L.S. Jeong, B. Pukall, J. Mello, A. Ratkowski and H.-H.
BURKE (1999): Atmospheric correction for shortwave spectral imagery based on MODTRAN4, SPIE Proc., 3753, 61-69.

AIG (2001): ACORN User's Guide, Stand Alone Version, Analytical Imaging and Geophysics (AIG), LLC, p. 64.

Berk, A., L.S. BERnSTEIN and D.C. Robertson (1989): MODTRAN: a moderate resolution model for LOWTRAN7, Rep. GL-TR-89-0122 (Air Force Geophys. Lab., Bedford, MA).

Cuomo, V., V. Tramutoli, N. Pergola, C. Pietrapertosa and F. Romano, (1997): In place merging of satellite based atmospheric water vapor measurements, Int. J. Remote Sensing, 18 (17), 3649-3668.

GAO, B.-C. and A.F.H. Goetz (1990): Column atmospheric water vapor and vegetation liquid water retrievals from airborne imaging spectrometer data, J. Geophys. Res. Atmos., 95 (D4), 3549-3564.

JedLoveC, G.J. (1990): Precipitable water estimation from high-resolution split window radiance measurements, J. Appl. Meteorol. , 29, 851-865.

KleEsPIES, T.J. and L.M. McMillin (1984): Physical retrieval of precipitable water using split window technique, in Preprints Conf. On Satellite Meteorology/Remote Sensing and Applications, AMS, Boston, 55-57.

Menzel, W.P. and L.E. GuMLEY (1998): MODIS Atmospheric profile retrieval - Algorithm Theoretical Basis Document, ATBD-MOD-07 (University of Wisconsin, Madison).

Ottle, C., S. Outalha, C. Francois and S. LeMaguer, (1997): Estimation of total atmospheric water vapor from split-window radiance measurements, Remote Sensing Environ., 61 (3), pp.410-418.

Tanré, D., C. Deroo, P. Duhaut, M. Herman, J.J. Morchrette, J. Perbos and P.Y. Deschamps (1986): Simulation of the Satellite Signal in the Solar Spectrum (5S), Users's Guide (U.S.T. de Lille, 59655 Villeneuve d'Ascq, France: Laboratoire d'Optique Atmospherique). 